\title{
Ventricular Tachycardia Unveiling an Obstructive Cardiomyopathy Complicated with an Apical Aneurysm
}

\author{
W. N. Mboup*, S. A. Sarr, D. W. Balde, M. T. Diop, Y. Diouf, J. S. Mingou, F. Aw, \\ M. Bodian, M. B. Ndiaye, M. Diao \\ Cardiology Department, Aristide Le Dantec University Hospital Center, Dakar, Senegal \\ Email: *walynmboup@yahoo.fr
}

How to cite this paper: Mboup, W.N., Sarr, S.A., Balde, D.W., Diop, M.T., Diouf, Y., Mingou, J.S., Aw, F., Bodian, M., Ndiaye, M.B. and Diao, M. (2022) Ventricular Tachycardia Unveiling an Obstructive Cardiomyopathy Complicated with an Apical Aneurysm. World Journal of Cardiovascular Diseases, 12, 111-117.

https://doi.org/10.4236/wjcd.2022.122011

Received: December 17, 2021

Accepted: February 22, 2022

Published: February 25, 2022

Copyright $(\odot) 2022$ by author(s) and Scientific Research Publishing Inc. This work is licensed under the Creative Commons Attribution International License (CC BY 4.0).

http://creativecommons.org/licenses/by/4.0/

\begin{abstract}
Background: We report the case of a 58-year-old hypertensive patient under treatment who presented with a ventricular tachycardia unveiling an obstructive cardiomyopathy complicated with an apical aneurysm. Aim: Highlight the rarity of the case and the difficulty of management. Case Presentation: This patient was transferred from Regional Hospital of Ziguinchor in southern Senegal for a brutal dizzy spell without loss of consciousness. The electrocardiogram showed a wide monomorphic QRS complex regular tachycardia with a rate of 215 beats $/ \mathrm{min}$. An external electrical cardio version at 300 joules was applied which led to the recovery of a sinus rhythm on the electrocardiogram. The Doppler echocardiography showed an asymetricalmedio-ventricular hypertrophy with a maximum left intra-ventricular gradient at $10 \mathrm{~mm} \mathrm{Hg}$ at rest. The coronarography via the radial artery was normal. The evolution was labeled with a recurrence of the ventricular tachycardia. The patient was then put on amiodarone $200 \mathrm{mg}$, beta-blocker (bisoprolol 10 $\mathrm{mg}$ ) and anti-vitamin $\mathrm{K}$ (acenocoumarol $4 \mathrm{mg}$ ). Facing rhythmic instability, an implantable automatic defibrillator was fitted. No complication has been reported after one year of evolution. Conclusion: HCM with medio ventricular obstruction and apical aneurysm complicated with ventricular arrhythmias is a rare entity. Its management is difficult and controversial.
\end{abstract}

\section{Keywords}

Hypertrophic Cardiomyopathy, Apical Aneurysm, Ventricular Tachycardia, Implantable Automatic Defibrillator

\section{Introduction}

Hypertrophic cardiomyopathy $(\mathrm{HCM})$ is the most common genetic heart disease 
with an estimated prevalence of $0.02 \%$ and $0.2 \%$ of the general population [1]. Its features are an unexplained usually asymmetric hypertrophy of the left ventricle, combined with undilated ventricular chambers in the absence of another cardiac or systemic disease capable of causing ventricular hypertrophy [2] [3].

In its medio-ventricular form, it can be complicated with an apical aneurysm.

Under-diagnosed, apical aneurysm throughout this heart condition is increasingly described [4].

These aneurysms are seen in $2 \%$ of HCM and may have prognostic implications.

They usually are the source of severe and potentially fatal arrhythmias and are generally the site of thrombus.

The aim is to highlight the rarity of the case and the difficulty of management.

\section{Observation}

It was about a 58-year-old hypertensive patient undergoing a treatment with a good follow-up. He had a sedentary lifestyle and no family history of sudden cardiac death.

He was transferred from Regional Hospital of Ziguinchor (Southern Sénégal) for a sudden onset discomfort with dizziness without loss of consciousness with simultaneous spontaneous and hyperkinetic palpitations of sudden onset as well, without pain nor dyspnea and it lasted for 10 minutes approximatively. This was an inaugural episode.

At his admission, he presented with a cardiovascular collapse with impregnable blood pressure, a thready and quick pulse, profuse sweating and intense pallor.

No sign of heart failure was found. The electrocardiogram showed a wide monomorphic QRS complex regular tachycardia with a rate of 215 beats/min without fusion beats nor capture beats as shown in Figure 1.

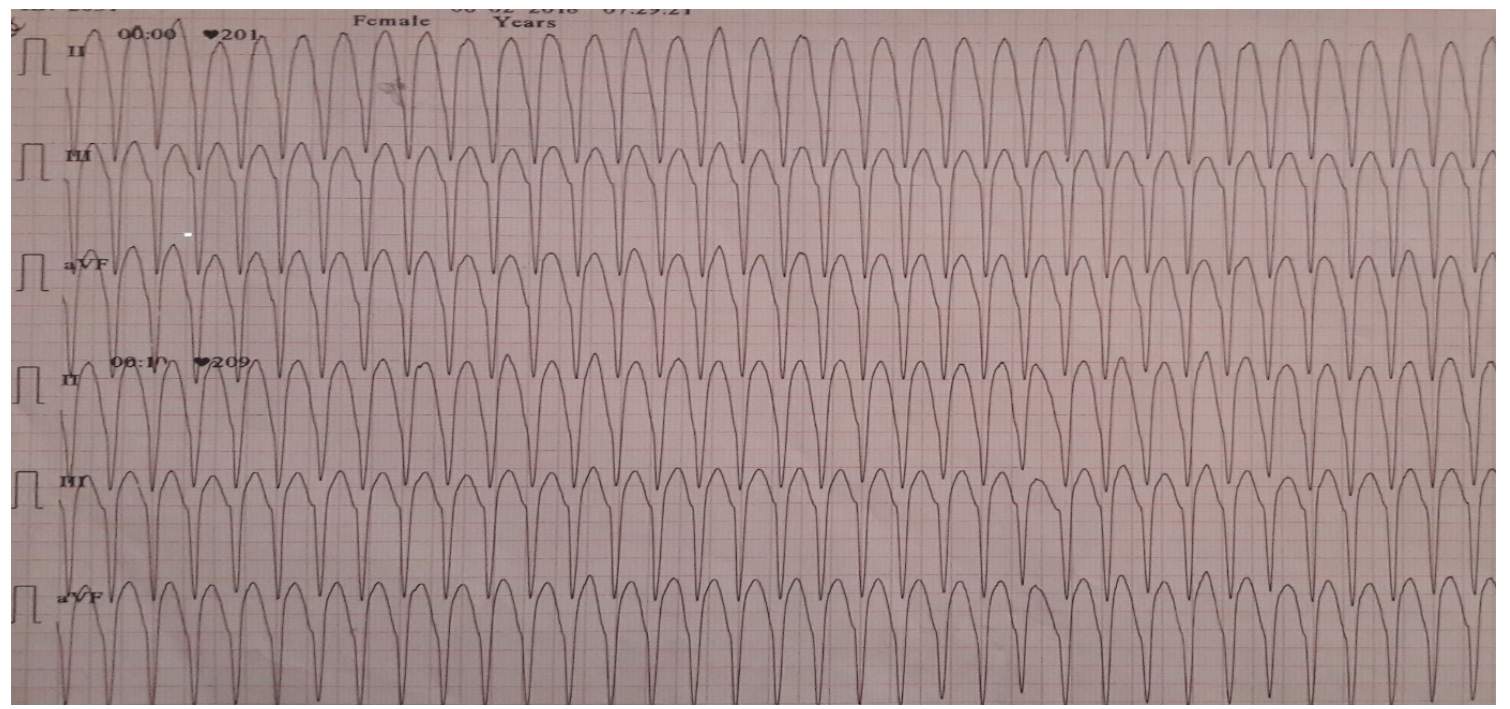

Figure 1. 12 leads EKG displaying a ventricular tachycardia with a rate of 215 beats/min. 
An external electrical cardioversion at 300 joules was applied which led to the recovery of a sinus rhythm on the electrocardiogram. Facing the recurrence of VT about ten minutes after the EECV, a loading dose of amiodarone was given followed with another EECV at 300 joules which led to the decrease of the VT.

The EKG then showed a sinus regular rhythm with a rate of 60 beats/min with, furthermore, a necrosis in septo-apico-lateral, rR' aspect in V1 V2 and a right atrial hypertrophy (Figure 2).

The Doppler echocardiography showed an asymetricalmedio-ventricular hypertrophy with a maximum left intra-ventricular gradient at $10 \mathrm{~mm} \mathrm{Hg}$ at rest (Figure 3). We also noticed an apical and aneurysmal distortion, site of an enclosed and poorly mobile thrombus (Figure 3 ) without mitral valve insufficiency. Both atria were dilated with a diastolic dysfunction and a restrictive profile.

The coronarography was normal (Figure 4).

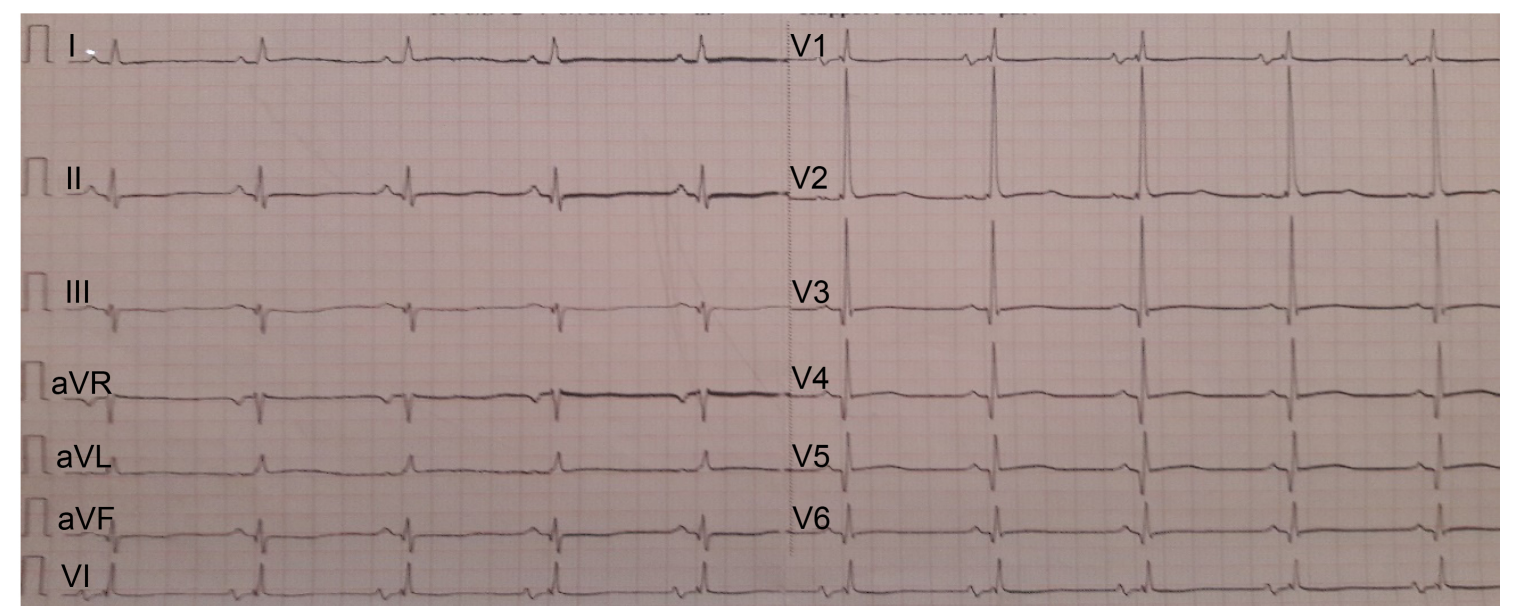

Figure 2. EKC showing a sinus regular rythm with a rate of 60 beats/min, a necrosis in septo-apico-lateral, rR' aspect in V1 V2 and a right atrial hypertrophy.

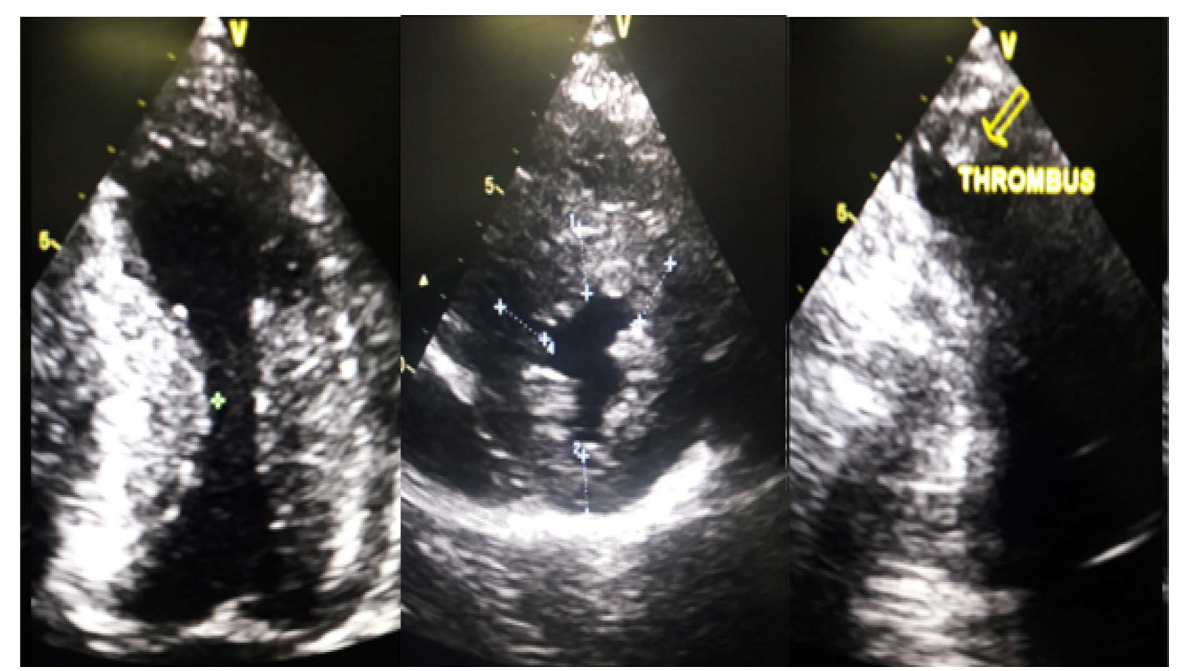

Figure 3. Echocardiographic images (4 chambers apical view centered on the left ventricle and transventricular minor axis) showing the hypertrophy and the medio-ventricular obstruction, the apical aneurysm and the thrombus within. 
Biological analysis including complete blood count, TSHus, blood ionogram, renal and inflammatory check-up, glycemia and retroviral serology were normal.

The evolution was labelled by a recurrence of the ventricular tachycardia after a week. In addition to amiodarone (firstly loading dose of $1800 \mathrm{mg}$ then $200 \mathrm{mg}$ per day 5 days a week) the patient received a betablocker (bisoprolol 10mg) and anti-vitamin K (acénocoumarol). Despite optimal medical treatment, the patient continued to exhibit rhythmic instability.

At the end, a double chamber implantable automatic defibrillator from Biotronik was fitted as illustrated in Figure 5.

No complication has been reported after one year of evolution.

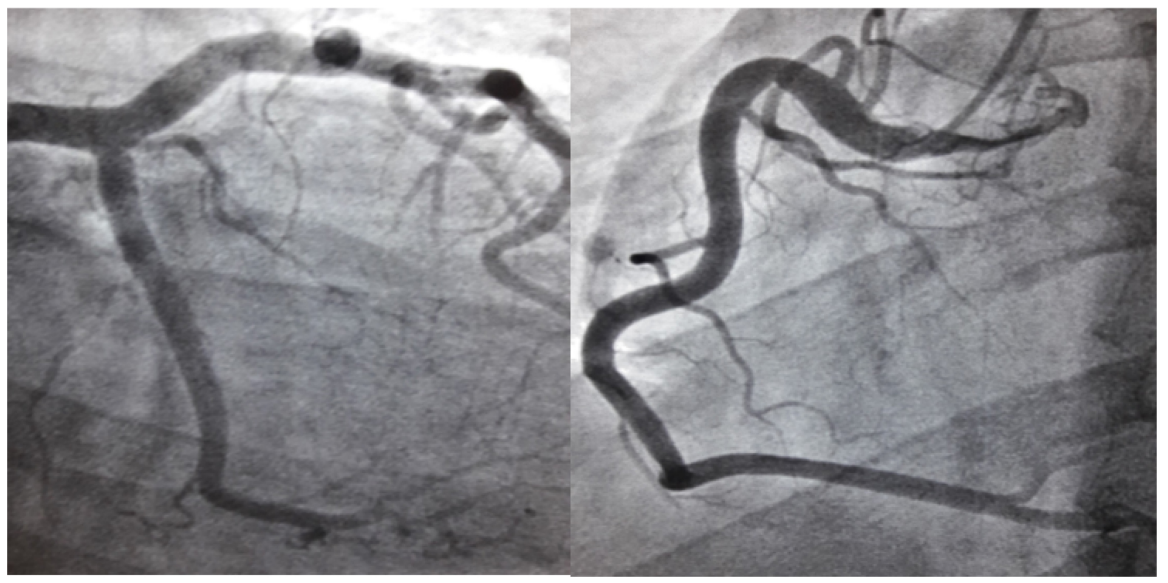

(a)

(b)

Figure 4. Coronarographic images showing left (a) and right (b) healthy coronary artery. Image A: incidence Spider; Image B: incidence OAG.

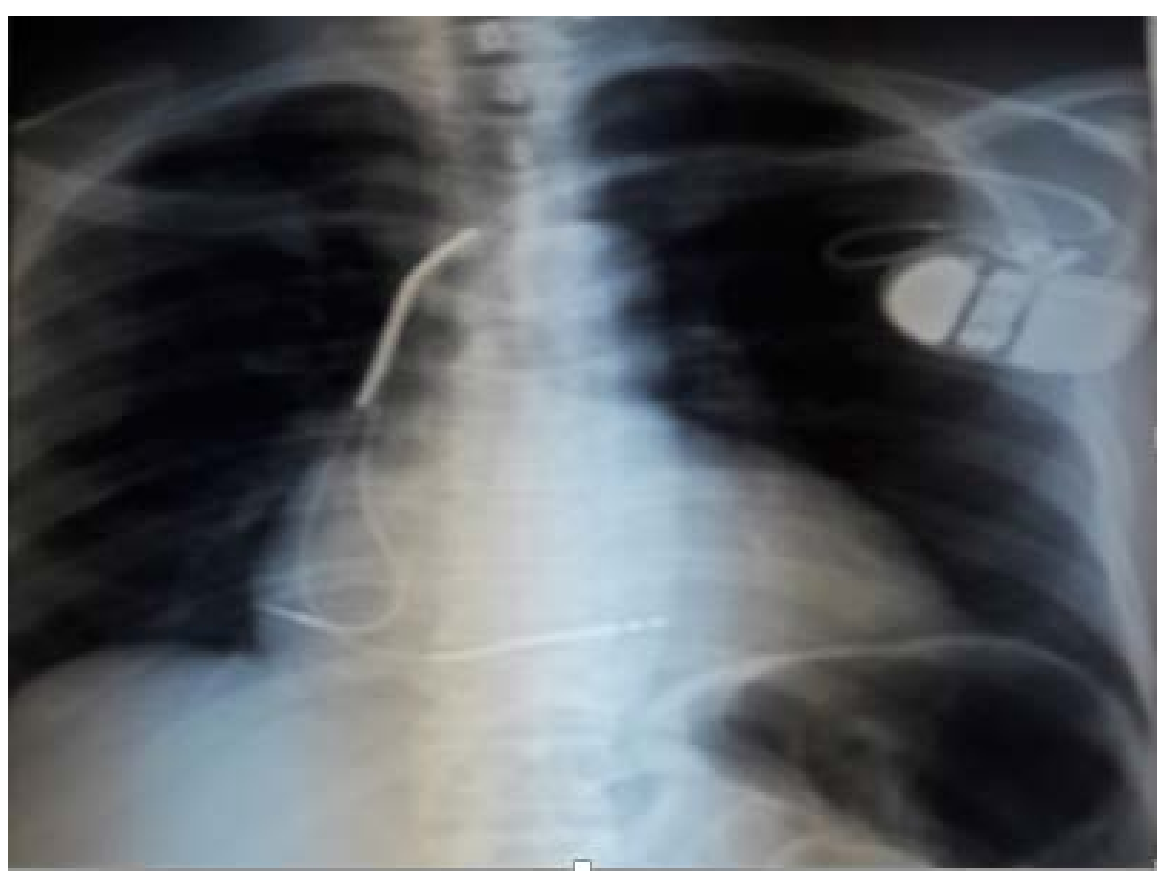

Figure 5. Chest X-ray showing a double chamber implantable automatic defibrillator. 


\section{Discussion}

Apical aneurysm is currently considered as a severe complication of HCM and is an arrythmia substrate exposing to potentially fatal arrythmias. These aneurysms are seen in 2\% of HCM [5] and can lead to prognostic implications.

They are the site of thrombi [6] as shown in our case. The particularity of this case lies in the fact that it is quite rare to see the following association: ventricular arrhythmia, medio-ventricular hypertrophic cardiomyopathy, aneurysm and apical thrombi. These ventricular arrhythmias expose to a risk of sudden cardiac death [7] [8].

Some authors rank patients presenting an apical aneurysm as being of high risk of sudden cardiac death; they also deem that the annual mortality rate may reach $10.5 \%$.

Given the existence of ventricular aneurysms due to a coronaropathy, a coronarography was done to exclude any coronary affection on our patient. The echocardiography is an important tool in the diagnostic of the HCM. The echocardiography check-up of an HCM must evaluate: the structure and the cardiac function, the dynamic obstruction, the mitral anomalys and the severity of the mitral insufficiency, the myocardial fibrosis if possible. In a first study about 423 patients and in another regarding 490 patients, authors showed that medio ventricular obstruction has a certain negative effect on the mortality rate by sudden cardiac death, in particular on its relations with fatal ventricular arrhythmias [7] [8].

However, the MRI still keeps its role on patients with poor echogenicity for whom, the echocardiographic exam can't be conclusive with a recommendation level IB. It allows, indeed, exacts measurements of the myocardial thickness and the ventricular mass [9] [10].

The management of an apical aneurysm complicating a medio ventricular HCM is very difficult and controversial. The aim of the drug treatment is to improve symptoms with a raise of functional capacity at exertion.

Empirically, drug treatment is started when some symptoms shows such as exertionaldyspnea, chest pain, dizziness, syncope and presyncope [11].

Classic drug treatments include betablockers, verapamil and disopyramide. A treatment with anti-vitamin $\mathrm{K}$ is widely prescribed because it is a very embolizing pathology.

When used at a low dose (less than $300 \mathrm{mg}$ ), amiodarone has shown an improvement on the survival of patients with arrythmias, but this treatment requires a close surveillance and can be not sanctioned at long term on young patients because of the cumulative toxicity [12]. When there is a high risk, we must consider fitting an implantable defibrillator.

Regarding secondary prevention, all recommendations are unanimous to recommend the fitting of an implantable defibrillator on all these patients for the risk of sudden cardiac death is more than $10 \%$ per year [13] [14] [15].

American recommendations keep only one risk factor of sudden cardiac death 
to recommend the implantation of a defibrillator when there is a first degree family history of sudden cardiac death or when the maximum left ventricular thickness exceeds $30 \mathrm{~mm}$, also in case of recent unexplained syncope [15].

\section{Conclusion}

HCM with medio ventricular obstruction and apical aneurysm complicated with ventricular arrhythmias is a rare entity. Its management is difficult and controversial. The defibrillator is an alternative when it comes to the prevention of sudden cardiac death.

\section{Conflicts of Interest}

The authors declare no conflicts of interest regarding the publication of this paper.

\section{References}

[1] Maron, B.J., Gardin, J.M., Flack, J.M., Gidding, S.S., Kurosaki, T.T. and Bild, D.E. (1995) Prevalence of Hypertrophic Cardiomyopathy in a General Population of Young Adults: Echocardiographic Analysis of 4111 Subjects in the CARDIA Study Coronary Artery Risk Development in (Young) Adults. Circulation, 92, 785-789. https://doi.org/10.1161/01.CIR.92.4.785

[2] Maron, B.J., Yeates, L. and Semsarian, C. (2011) Clinical Challenges of Genotype Positive (+)-Phenotype Negative (-) Family Members in Hypertrophic Cardiomyopathy. American Journal of Cardiology, 107, 604-608. https://doi.org/10.1016/j.amjcard.2010.10.022

[3] Maron, B.J. and Semsarian, C. (2010) Emergence of Gene Mutation Carriers and the Expanding Disease Spectrum of Hypertrophic Cardiomyopathy. European Heart Journal, 31, 1551-1553. https://doi.org/10.1093/eurheartj/ehq111

[4] Rowin, E.J., Maron, B.J., Haas, T.S., Garberich, R.F., Wang, W., Link, M.S., et al. (2017) Hypertrophic Cardiomyopathy with Left Ventricular Apical Aneurysm: Implications for Risk Stratification and Management. Journal of the American College of Cardiology, 69, 761-773. https://doi.org/10.1016/j.jacc.2016.11.063

[5] Maron, M.S., Finley, J., Bos, J.M., Hauser, T.H., Manning, W.J., Haas, T.S., et al. (2008) Aneurysms in Hypertrophic Cardiomyopathy. Circulation, 118, 1541-1549. https://doi.org/10.1161/CIRCULATIONAHA.108.781401

[6] Olszewski, R., Timperley, J., Szmigielski, C., Monaghan, M., Nihoyannopoulos, P., Senior, R., et al. (2007) The Clinical Applications of Contrast Echocardiography. European Journal of Echocardiography, 8, S13-S23. https://doi.org/10.1016/j.euje.2007.03.021

[7] Minami, Y., Kajimoto, K., Terajima, Y., Yashiro, B., Okayama, D., Haruki, S., et al. (2011) Clinical Implications of Midventricular Obstruction in Patients with Hypertrophic Cardiomyopathy. Journal of the American College of Cardiology, 57, 2346-2355. https://doi.org/10.1016/j.jacc.2011.02.033

[8] Efthimiadis, G.K., Pagourelias, E.D., Parcharidou, D., Gossios, T., Kamperidis, V., Theofilogiannakos, E.K., et al. (2013) Clinical Characteristics and Natural History of Hypertrophic Cardiomyopathy with Midventricular Obstruction. Circulation Journal, 77, 2366-2374. https://doi.org/10.1253/circj.CJ-12-1561

[9] Maron, M.S., Maron, B.J., Harrigan, C., Buros, J., Gibson, C.M., Olivotto, I., et al. 
(2009) Hypertrophic Cardiomyopathy Phenotype Revisited after 50 Years with Cardiovascular Magnetic Resonance. Journal of the American College of Cardiology, 54, 220-228. https://doi.org/10.1016/j.jacc.2009.05.006

[10] Olivotto, I., Maron, M.S., Autore, C., Lesser, J.R., Rega, L., Casolo, G., et al. (2008) Assessment and Significance of Left Ventricular Mass by Cardiovascular Magnetic Resonance in Hypertrophic Cardiomyopathy. Journal of the American College of Cardiology, 52, 559-566. https://doi.org/10.1016/j.jacc.2008.04.047

[11] Maron, B.J. (1997) Hypertrophic Cardiomyopathy. Lancet, 350, 127-133. https://doi.org/10.1016/S0140-6736(97)01282-8

[12] Maron, B.J. (2002) Hypertrophic Cardiomyopathy: A Systematic Rewiew. JAMA, 287, 1308-1320. https://doi.org/10.1001/jama.287.10.1308

[13] Elliott, P.M., Anastasakis, A., Borger, M.A., Borggrefe, M., Cecchi, F., Charron, P., et al. (2014) 2014 ESC Guidelines on Diagnosis and Management of Hypertrophic Cardiomyopathy: The Task Force for the Diagnosis and Management of Hypertrophic Cardiomyopathy of the European Society of Cardiology (ESC). European Heart Journal, 35, 2733-2779. https://doi.org/10.1093/eurheartj/ehu284

[14] Dubourg, O., Charron, P., Desnos, M., et al. (2011) Cardiomyopathie Hypertrophique: Protocole national de diagnostic et de soins pour les maladies rares. HAS. http://www.has-sante.fr/portail/jcms/c 1100272/fr/ald-n5-cardiomyopathie-hypertr ophique

[15] Gersh, B.J., Maron, B.J., Bonow, R.O., Dearani, J.A., Fifer, M.A., Link, M.S., et al. (2011) 2011 ACCF/AHA Guideline for the Diagnosis and Treatment of Hypertrophic Cardiomyopathy: A Report of the American College of Cardiology Foundation/American. Circulation, 124, e783-e831. https://doi.org/10.1161/CIR.0b013e318223e2bd 